2010 Octubre, 2(1): 1-

\title{
LIVER FATTY ACID BINDING PROTEIN (LFABP) TRANSFERS FATTY ACIDS AND FATTY ACYL COAS TO MEMBRANES
}

\author{
Autores \\ Eduardo De Gerónimo\#+, Robert M. Hagan§, David C. Wilton§, and Betina Córsico\#+. \\ Lugar de Trabajo \\ \# INIBIOLP (CONICET-UNLP), University of La Plata, Calle 60 y120, 1900, La Plata, Argentina. \\ +Consejo Nacional de Investigaciones Científicas y Técnicas, Avda. Rivadavia 1917, C1033AAJ, Ciudad. \\ Autónoma de Buenos Aires, Argentina. \\ $\S$ School of Biological Sciences, University of Southampton, Bassett Crescent East, Southampton SO16 7PX, \\ United Kingdom. \\ e-mail: bcorsico@atlas.med.unlp.edu.ar
}

\section{Introducción}

Liver fatty acid binding protein (LFABP) is a member of a family of structurally related small cytosolic lipid binding proteins. Fatty acid binding proteins (FABPs) are presumably involved in the uptake and targeting of long chain fatty acids (LCFA) to intracellular organelles and metabolic pathways, although their physiological functions are as yet unclear. Unlike other FABPs, LFABP binds not only LCFAs but also a wide range of other hydrophobic ligands, among them acyl CoAs, and this could have important physiological significance.

\section{Objetivos}

The objective of this work was to analyze LFABP's capacity to transfer acyl CoAs to artificial membranes and compare it to LCFA transfer employing natural ligands, in order to better understand the specific physiological role of LFABP in the cell.

\section{Materiales y métodos}

We have employed a pair of tryptophan containing mutants at position 28 (L28W) and 74 (L74W), whose fluorescence is modified upon LCFA and acyl CoA binding. This gives us the chance to study both the ligand-protein interaction and protein-to-membrane ligand transfer properties using physiological ligands instead of the analogues we have been employing previously.

\section{Resultados}

So far, our results indicate that the mutant proteins bind and transfer LCFA as well as acyl CoA to artificial membranes, under physiological conditions. LFABP seems to employ a diffusional mechanism of ligand transfer to membranes. However, the rates of transfer are markedly different. We have also observed that different acyl CoAs show specific partitioning and transfer characteristics.

\section{Conclusiones}

The ability of acyl CoA to compete with fatty acids for binding to LFABP and transfer from LFABP to membranes, further highlights the role of fatty acyl CoA in modulating LFABP function in the cell. 